\title{
Developing a computational ontology to understand the relational aspects of audience formation [version 1; peer
}

\section{review: 2 approved]}

\author{
Matthew Hanchard (D1, Peter Merrington1, Bridgette Wessels' ${ }^{1}$, Kathy Rogers2 \\ Michael Pidd ${ }^{2}$, Simeon Yates ${ }^{3}$, David Forrest ${ }^{4}$, Andrew Higson (iD) 5 , \\ Nathan Townsend ${ }^{5}$, Roderik Smits ${ }^{6}$ \\ ${ }^{1}$ School of Social and Political Sciences, University of Glasgow, Glasgow, Glasgow, G12 8RT, United Kingdom \\ ${ }^{2}$ Digital Humanities Institute, University of Sheffield, Sheffield, South Yorkshire, S3 7QY, United Kingdom \\ ${ }^{3}$ Department of Communication and Media, University of Liverpool, Liverpool, Merseyside, L69 7ZG, United Kingdom \\ ${ }^{4}$ School of English, University of Sheffield, Sheffield, South Yorkshire, S3 7RA, United Kingdom \\ ${ }^{5}$ Department of Theatre, Film, Television and Interactive Media, University of York, York, North Yorkshire, YO10 5DD, United \\ Kingdom \\ ${ }^{6}$ Filmuniversität Babelsberg Konrad Wolf, Potsdam, Brandenburg, 14482, Germany
}

V1 First published: 12 Feb 2020, 2:5

https://doi.org/10.35241/emeraldopenres.13465.1

Latest published: 12 Feb 2020, 2:5

https://doi.org/10.35241/emeraldopenres.13465.1

\section{Abstract}

In this article, we discuss an innovative audience research methodology developed for the AHRC-funded 'Beyond the Multiplex: Audiences for Specialised Film in English Regions' project (BtM). The project combines a computational ontology with a mixed-methods approach drawn from both the social sciences and the humanities, enabling research to be conducted both at scale and in depth, producing complex relational analyses of audiences. BtM aims to understand how we might enable a wide range of audiences to participate in a more diverse film culture, and embrace the wealth of films beyond the mainstream in order to optimise the cultural value of engaging with less familiar films. BtM collects data through a threewave survey of film audience members' practices, semi-structured interviews and film-elicitation groups with audience members alongside interviews with policy and industry experts, and analyses of key policy and industry documents. Bringing each of these datasets together within our ontology enables us to map relationships between them across a variety of different concerns. For instance, how cultural engagement in general relates to engagement with specialised films; how different audiences access and/or share films across different platforms and venues; how their engagement with those films enables them to make meaning and generate value; and how all of this is shaped by national and regional policy, film industry practices, and the decisions of cultural intermediaries across the fields of film production, distribution and exhibition. Alongside our analyses,

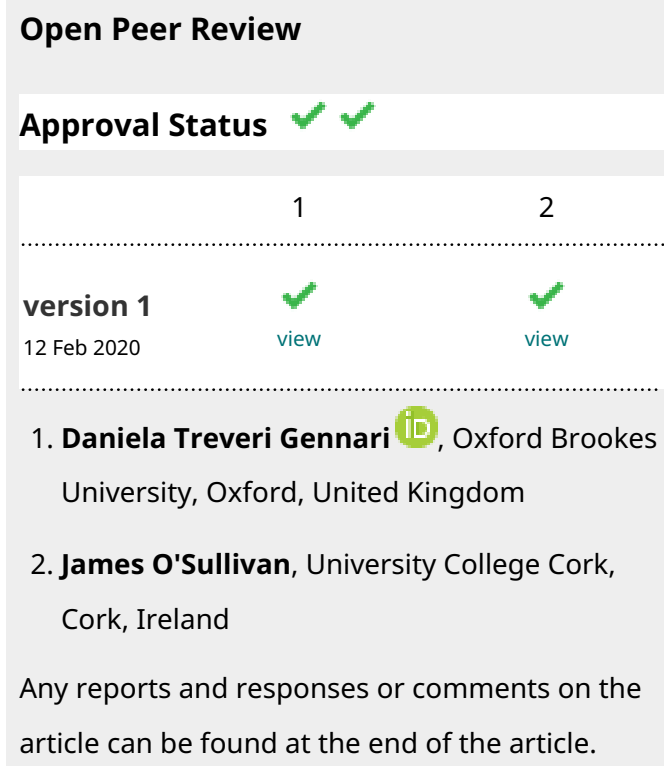

1 2

version 1

12 Feb 2020

view

1. Daniela Treveri Gennari (D), Oxford Brookes University, Oxford, United Kingdom

2. James O'Sullivan, University College Cork,

Cork, Ireland

Any reports and responses or comments on the article can be found at the end of the article. 
the ontology enables us to produce data visualisations and a suite of analytical tools for audience development studies that stakeholders can use, ensuring the research has impact beyond the academy. This paper sets out our methodology for developing the BtM ontology, so that others may adapt it and develop their own ontologies from mixed-methods empirical data in their studies of other knowledge domains.

\section{Keywords}

Audience studies, mixed-methods research, computational ontology, digital humanities

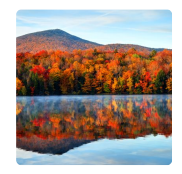

This article is included in the Fairer Society

gateway.

\section{Corresponding author: Matthew Hanchard (matthew.hanchard@glasgow.ac.uk)}

Author roles: Hanchard M: Conceptualization, Data Curation, Formal Analysis, Methodology, Resources, Writing - Original Draft Preparation, Writing - Review \& Editing; Merrington P: Conceptualization, Data Curation, Formal Analysis, Methodology, Resources, Writing - Original Draft Preparation, Writing - Review \& Editing; Wessels B: Conceptualization, Formal Analysis, Funding Acquisition, Investigation, Project Administration, Supervision, Writing - Original Draft Preparation; Rogers K: Resources, Software, Visualization; Pidd M: Conceptualization, Funding Acquisition, Methodology, Project Administration, Supervision, Visualization; Yates S: Formal Analysis, Resources, Visualization; Forrest D: Formal Analysis, Funding Acquisition; Higson A: Formal Analysis; Townsend N: Formal Analysis; Smits R: Formal Analysis

Competing interests: No competing interests were disclosed.

Grant information: This work is supported by the UK Arts and Humanities Research Council (AHRC) under grant reference AH/P005780/1. The data and analysis presented in this paper are part of the AHRC funded project 'Beyond the Multiplex: Audiences for specialised film in English regions' [AH/FP005780/2F1].

The funders had no role in study design, data collection and analysis, decision to publish, or preparation of the manuscript.

Copyright: @ 2020 Hanchard $\mathrm{M}$ et al. This is an open access article distributed under the terms of the Creative Commons Attribution License, which permits unrestricted use, distribution, and reproduction in any medium, provided the original work is properly cited.

How to cite this article: Hanchard M, Merrington $P$, Wessels $B$ et al. Developing a computational ontology to understand the relational aspects of audience formation [version 1; peer review: 2 approved] Emerald Open Research 2020, 2:5

https://doi.org/10.35241/emeraldopenres.13465.1

First published: 12 Feb 2020, 2:5 https://doi.org/10.35241/emeraldopenres.13465.1 


\section{Introduction: Audiences for specialised film in English Regions}

This paper discusses an innovative audience studies research methodology developed through the Arts and Humanities Research Council (AHRC)-funded project, 'Beyond the Multiplex: Audiences for Specialised Film in English Regions' (UKRI, 2017), using mixed-methods research and a computational ontology. The methodology for Beyond the Multiplex (BtM from here on) addresses one of the key challenges of audience studies research: how to capture the full richness of audiences' experiences within the wider context of cultural provision and access. Our use of a computational ontology (ontology here on) enables us to interrogate a diverse range of datasets, and to map relationships between the constituent elements of audiences and their experiences of specialised film. That is, our approach allows us to address questions about: how cultural engagement in general relates to engagement with specialised films; how different audiences access different types of films across different platforms and venues; how their engagement with those films enables them to make meaning and generate value; and how all of this is shaped by national and regional policies, industry practices and the decisions of cultural intermediaries across the fields of production, distribution and theatrical and online exhibition.

The paper starts with an overview of the BtM project, including an outline of its research design and an explanation of how the ontology was initially developed, and how it has evolved iteratively through the addition of various datasets (interviews, surveys, film-elicitation groups, and analyses of policy and industry documents). We then set out each of the datasets separately, to explain how the data was gathered, its structure and format, and how it was incorporated into the ontology. In the penultimate results section, we explain how these separate datasets are brought together in the ontology through illustrative examples. We then conclude by arguing that other researchers who adopt and adapt our approach may similarly explore large mixed-methods datasets in detail and at scale.

\section{About the project}

The BtM project aims to understand and assess how audiences form in relation to specialised film within four English regions (the North East, North West, South West, and Yorkshire and the Humber). Specifically, we seek to understand: (a) how to enable a wider range of audiences to participate in a more diverse film culture that embraces the wealth of films beyond the mainstream; and (b) how to optimise the cultural value of engaging with less familiar films. We use the concept of 'specialised film' because it is widely used in film trade circles, and until recently in official film policy documents, to refer to any type of nonmainstream film. The category includes small-scale UK films, foreign language ones, documentaries, archival films, and films with unconventional narratives, themes or cinematic techniques. We acknowledge that 'specialised' is not a fully accurate term, with our research showing that many in the sector would prefer an alternative. We also find that most audience members use alternative labels, such as art-house, or independent. However, for the sake of consistency across our datasets (including the analysis of industry and policy documents), we use the term 'specialised film' throughout this paper.

Our methodology employs an ontology to model and analyse diverse datasets and their relationships to one another. In this, data from interviews, film-elicitation groups, surveys, secondary analysis of statistical data, and document analyses are brought together to understand the meaning, experiences, and value of film for audiences, as well as capturing wider trends such as the socio-cultural profiles of different audiences and how film policy and industrial practice impacts on their access to films. The ontology enables us to interrogate those diverse datasets simultaneously and consistently, and to generate various analytical tools and data visualisations. Our aim is to make these tools and visualisations publicly available to audience development stakeholders, so that they may use them freely for their own research and decision-making, increasing the significance and impact of our work beyond the academy.

\section{Designing mixed-methods audience research}

There is a long history of research about film and television audiences, going back to the 1920s. The methodologies used to undertake audience studies research, and the conclusions drawn about audience behaviours, have changed over time in various ways too (Livingstone, 2018). However, there are long-standing tendencies throughout. For example, contemporary audience studies research often involves either large-scale quantitative surveys to capture broad behaviours and trends (e.g. Arts Council England, 2011), or much smaller qualitative studies of specific audience experiences (e.g. Evans, 2011). While such studies clearly have many strengths and merits, they also harbour weaknesses. For example, findings from qualitative studies cannot be generalised, while quantitative arts impact studies often hold positive bias and tend to overstate the impact on audiences of particular art forms (Johanson \& Glow, 2015).

The significance of these weaknesses is highlighted when we recognise audience experiences as rich and diverse (Barker \& Mathijs, 2012; Christie, 2012) and/or hold audiences as relational and interactive (Corbett \& Wessels, 2017; Livingstone, 1998). These insights suggest that research needs to address the relationships that audiences have with film, including how they watch them - both online, at events and festivals, and at cinemas. Also, how audiences might interpret film narratives and content, and how significant place, venues and events are for their experience of film, and what impact film policy and industry practice has on real and perceived levels of access and provision. Furthermore, because access to specialised film is uneven across the Britain, research needs to address the local and regional circumstances of film audiences. To address these questions, we adopt a mixed methods approach. This enables us to understand the richness of audience experiences through qualitative methods, and to identify broader trends in audiences' film-watching activities through quantitative methods. By combining both approaches we gain a better understanding of the increasingly diverse ways that people come together to form film audiences, and the depth, 
variety and richness of audience experiences they encompass, which we might not have otherwise gained by taking just one approach. To combine our qualitative and quantitative data we follow Crossley \& Edward's (2016) argument that mixed-methods researchers should be attentive to the ways in which each dataset frames a specific research focus. By coding data systematically, we explore the relations between data - both within the same dataset, and across different datasets (Cresswell, 2009). This is where the ontology comes in, since it is designed to help us analyse the data and the relations between data in a highly systematic manner.

To conceptualise these relations, we use Becker's (1982) notion of 'art worlds' to highlight the significance of relations between producers, distributors and consumers (Merrington et al., 2019). By applying this to film, we explore the relations between different 'film worlds'. In this, our film worlds are composed of film industry leaders, policy-makers and filmmakers; distributors, online platform operators and other cultural intermediaries and gatekeepers; broadcasters, festival organisers, venue managers and programmers; marketing people and film critics; and various audiences. The activities of each world, and how they relate to one another, generates particular audience experiences within the wider context of policy development, film industry practices and cultural consumption. Employing the concept of 'film worlds' and articulating it through our ontology allows us to examine film audiences in a complex and relational way that considers specific film audience formations alongside the broader circumstances in which those audiences form.

BtM addresses the provision of (and engagement with) specialised film in four English regions (the North East, North West, South West and Yorkshire and the Humber). Each of which have low levels of provision compared with London (BFI, 2012; BFI, 2017). We examine film provision and consumption in theatrical, place- and venue-based exhibition, including multiplexes, independent cinemas, film clubs and community cinema, as well as through events such as film festivals. We also address non-theatrical forms of film provision and consumption such as television, DVD, Blu-Ray and the various online/on-demand platforms. To further address the question of provision, we also examine the ways in which films are made available to audiences through the work of the funding and distribution gatekeepers who determine what sorts of films get made, and which of them are put into distribution, and under what conditions. We also look at audiences themselves, and their experiences of specialised films.

The project received ethical approval from the Newcastle University Faculty of Humanities and Social Science (HaSS) Research Ethics Committee (REC) in 2017, under reference BH161701 prior to project transferral into the University of Glasgow in 2018. Overall, to gather our data the project used the following methods:

- 200 semi-structured interviews with film viewers to understand film viewing and audience practices (see Wessels et al., $2019^{1}$ for a copy of the questions, anonymised transcripts, and NVivo coding scheme).
- Secondary analysis of data from existing surveys (See Yates et al. (2019b) and Hanchard et al. (2019) and carried out our own three-wave survey with audience members to develop a set of socio-cultural profiles for film audiences and to examine their patterns of consumption and Yates et al., 2019a).

- 16 film-elicitation groups to explore how audiences make sense of specialised film narratives (see Forrest et al., 2019).

- Analysis of 114 film policy documents (see Higson et al., 2020b) and interviewed 27 film policy and industry experts (see Higson et al., 2020b) to understand the industry context in which film-viewing takes place.

In combination, these methods generated a rich array of qualitative and quantitative data, providing insights about general patterns of film consumption, the contexts in which it takes place, and the social networks that enable it. They also provide rich experiential accounts of how audience members engage with a range of films across various screens, venues, and platforms. To understand how these combine to form different film worlds, we developed our ontology to bring all the data together, and in doing so automated the mapping of complex interrelationships between them - and thus between the constituent elements of various film worlds. In this paper we use pseudonyms throughout, this provides anonymity for participants in each dataset, and for ease of reading in outputs compared to participant codes or references.

\section{Defining an ontology in mixed-methods audience studies}

In computational terms, an ontology is a data model that describes the components, characteristics and interrelationships of a particular knowledge domain. Our ontology (Figure 1) describes the knowledge domain of film, audiences, and film industry policy through three classes of information: (1) entities (2) entity characteristics (3) and the relationships between them. For example, film is an entity which has characteristics such as specific actors and/or characters, a genre, a plot, a title, and a duration (how many minutes the film lasts). It also holds relationships with other entities, e.g. the entity film is related to other entities such as actor and venue - each of which hold their own set of characteristics. In this, relationships between various entities and characteristics hold their own set of values which can explicitly be named. For example, the two entities film and cinema are connected by a relationship called is watched at. This helps to identify which film genres are watched at the cinema. That is, film and cinema are mapped as entities, genre is mapped as a characteristic associated with film, and is watched at is held as a named relationship between film and cinema. While researchers can generate similar connections between data manually, the ontology allows vast

${ }^{1}$ In some interviews, two people were interviewed at the same time and place (e.g a married couple) - as such there are 197 transcripts vs. 200 interviews - as noted in the classification sheet (see Wessels et al., 2019). 


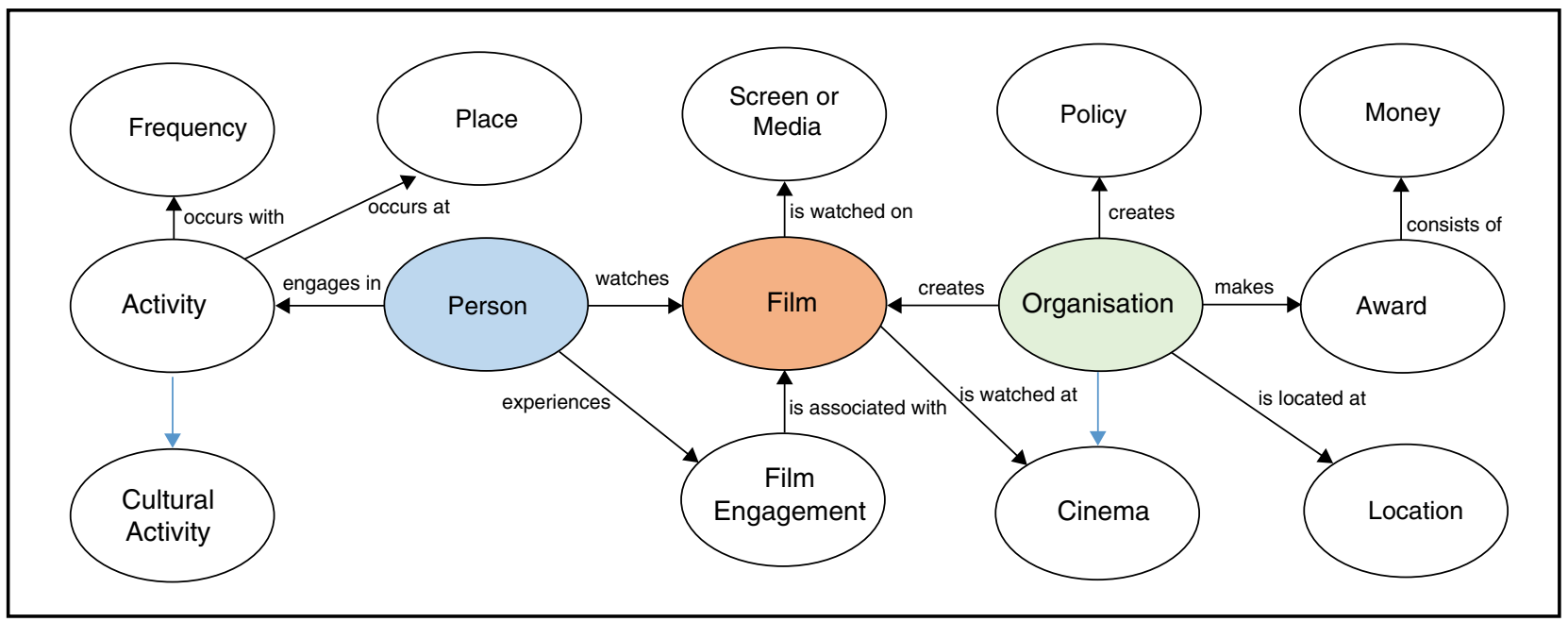

Figure 1. Schematic diagram of entities, characteristics, and relationships within our ontology.

quantities of data to be systematically processed to do this automatically in a way that is far beyond the capabilities of a research team (see Results section below).

It is important to understand that an ontology is a conceptual data model - it is not data, nor is it a database. An ontology is data agnostic; it defines a knowledge domain rather than types of data, and so it can accommodate data of varying types. Data models are used to define how data should be described, structured, and related. For instance, in our project we used the ontology to define how we structured a set of tables in a MySQL database. Later, we developed this into a more refined graph database using the programming language Java, making sure that its structure remained in line with the rules of the ontology. In this, our approach differs from research that uses relational data modelling (e.g. to develop traditional relational databases). While the latter enable two named entities to be connected to one another, they provide no details about the relationship in itself. For example, a relational model might show that an entity named Film is connected to another named Venue in a dataset, but it would not enable a researcher to differentiate between the film being shown at the venue, produced there, or the venue appearing as a filming location within the plot of a film. As a result, the structure of relational databases require that users manually infer the relationships between entities. An ontology goes beyond this by explicitly defining the relationships between entities and entity characteristics in the data. For example, in our ontology the entities film and venue are connected by a relationship called shown at (see Figure 1). In this, the relationships between entities and/or entity characteristic are treated as data. In information science, this type of model (composed of a tri-part structure of entity, characteristics, and relationship) is often referred to as either a 'triple' or 'subject-predicate-object'. Later in this paper, we explain how the ontology supports our data analysis in more detail (see Results section below) using a WebVOWL diagram and primary empirical data to clarify how the triples within our ontology enable us to develop arguments about film consumption and audiences in ways that would not be possible through standalone analyses of individual datasets or through conventional relational data modelling.

As we have noted elsewhere (see Pidd \& Rogers, 2018), two of the entities at the centre of our ontology (Film and Person) relate to relationships between films and their audiences (see Figure 1). Another entity, called Organisation is important because it associates both films and audiences with the organisations that screen or stream films, or organise film-related events, and with the organisations that shape film policy. In this, the ontology shows that a Person may have a relationship a particular Location (e.g. venue or town) and/or Film (e.g. genre), and specific types of Audience(s) when watching alongside having relationships with other types of Cultural activity (e.g going the theatre, or attending an art gallery). In addition, various experiences are captured as entity characteristics associated with Film - such as Motivation, Memory, and Response (e.g. a person's response to watching a film). This type of structure enables us to link an individual person to both qualitative and quantitative data, allowing us to assess their behaviour with respect not only to film consumption, but also to other cultural activities, either to be viewed within an individual context or as part of a larger socio-economic group.

The way we structure data to make it ready for ingestion into our graph database is aligned with the ontology. Statistical data can be mapped to the ontology's structure directly with measures and variables consistent across the dataset (see the section on 'Developing and analysing surveys' below). However, qualitative data such as interview and film-elicitation group transcripts, and policy and industry document analyses 
require manually processing. To process this unstructured data we use QSR's qualitative data analysis software package NVivo (Pro edition, version 11). Manually coding qualitative data is a labour-intensive process, requiring close reading and interpretation on the researchers' part. It constitutes a first stage of qualitative analysis which informs our understanding of audiences and enables us to evolve the ontology to better reflect the characteristics of the evidence (see the section on 'Audience interviews and coding' below).

When analysed separately, each dataset presents a window onto just one aspect of specialised film audiences (one film world). The ontology, by contrast, enables us to develop a holistic account, by modelling and interrelating all our datasets consistently, irrespective of their original format or type. By allowing us to directly compare different datasets, and to expand on the relationships between them, the ontology provides a means of analysing data both in-depth and at scale. That is, it allows us to identify broad patterns in the way audiences form, as well as delving deeply into the richness and diversity of people's experiences through their formation. In the sections that follow, we discuss various types of research we have undertaken, and the data generated for the ontology in each of them.

\section{Audience interviews and coding}

To understand how people engage with specialised film, we undertook 50 semi-structured interviews in each of our four studied English regions (North East, North West, South West, and Yorkshire and the Humber) between November 2017 and April 2018. We took a snowball sampling approach to recruitment, which provided a diverse sample of interviewees (see classification sheet in Wessels et al., 2019) split evenly between female $(48.02 \%)$ and male $(52.90 \%)$ and $<1 \%$ 'other'; $12.87 \%$ were from a black or minority ethnic (BAME) background; $68.32 \%$, were in some form of employment; $72.46 \%$ held an undergraduate degree or equivalent qualification; $15.35 \%$ had some form of disability; with ages distributed relatively evenly across the following groups 18-24 (15.84\%), 25-34 (26.24\%), 35-44 (12.87\%), 45-54 (16.34\%), 55-64 (15.35\%), and 65+ (15.35\%). Participants were identified through their membership of organisations, local interest groups (including non-film related ones, such as the University of the Third Age and running clubs), cinema film clubs, and through their attendance of film festivals. This often led to further participants being recommended to us, and at times for people to contact us themselves (snowball sampling). We recruited participants in several ways, contacting organisations either by telephone, by email, or by arranging to meet a key stakeholder in person - either in order to interview them, or to request they distribute a request for participants on our behalf. The interviews were then carried out in various places, based on the interviewee's preference (they chose the site) or a specific cinema site (where appropriate). The diversity of our sample meant that we could explore how people engage with both mainstream and specialised films in order to gain a better understanding of the cultural value people place on their engagement with each, and the barriers that inhibit such engagement.
We used a topic guide with a suggested set of questions (see interview questions in Wessels et al., 2019) as a way to facilitate consistency and thematic focus across interviews (Silverman, 2010). While the topic guide structured the sequence in which we asked questions, it remained loose enough to allow interviewees to speak freely and openly, thus maintaining a degree of naturalism in each interaction. The topic guides allowed us to ask participants a sequentially ordered set of questions about their film preferences, where and how they watch films, and with whom. We also explored how they were introduced to specialised film, whether their viewing habits have changed over time, and how they choose which films to watch. We closed with a broad set of questions on people's perception of audiences, the cultural value placed on film in general, and its perceived importance relative to other cultural activities.

To enable detailed analyses of the interview data, we carried out a process we call 'dual coding' - which entails qualitatively coding interview transcripts both descriptively and conceptually. In our descriptive qualitative coding, we started with an initial set of high-level codes derived from an initial iteration of the ontology; we then developed a hierarchy of codes beneath each high-level one, using descriptive terminology to name them (Saldana, 2012). In our conceptual qualitative coding, we used a combination of descriptive codes, 'in vivo' codes '...rooted [verbatim] in the participant's own language' (Saldana, 2012, p. 105), and gerund verb-based codes (Charmaz, 2015). For example, beneath a high-level, ontology-derived descriptive qualitative code called 'Times', we developed a set of descriptive ones that encompass characteristics such as the 'Time of day' a film was watched, and the specific 'Day of week' or 'Time of year' (Figure 2). At the same time, we also developed a set of conceptual qualitative codes to encompass specific 'Life stages' ranging from 'Childhood' and 'Pregnancy' through to interviewee-defined physiological and affective states, such as 'Busy with work', 'Down/depressed', 'Hungover', 'Ill or sick', or 'Being lazy'.

Our qualitative coding of interviews produced a coding scheme (see NVivo coding scheme in Wessels et al., 2019) with a complex hierarchy that informed a revision to the ontology. As such, we found that 'dual coding' generates a rich set of codes for analysis that allow us to address consumption patterns, to explore audience members' experiences and understandings of film, and to connect both with broader trends in production and distribution through the ontology, as evidenced through our analysis of documents relating to policy and industry practice.

\section{Developing and analysing surveys}

To identify national and regional patterns in how audiences form around film we conducted a three-wave survey over a six-month period and undertook secondary analysis of two existing survey datasets. The three-wave survey was delivered in collaboration with the Audience Agency using a nationally representative online panel from Research Now for recruitment. The first wave was conducted in August 2018 (gathering 5071 responses), with the second and third waves conducted 


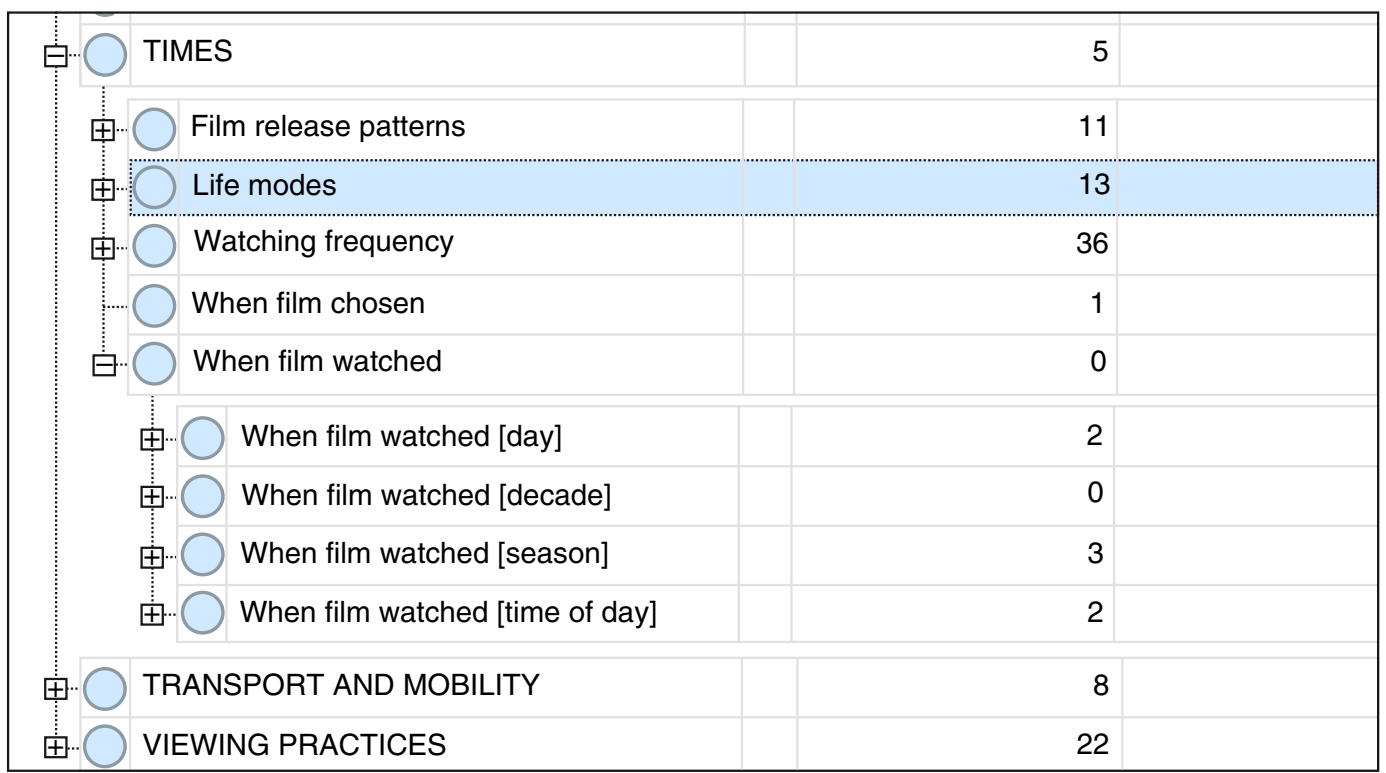

Figure 2. NVivo image of hierarchical ordered codes (expanded on Times).

in October 2018 and January 2019 (gathering $\mathrm{n}=547$ and $\mathrm{n}=250$ responses respectively).

The two secondary datasets were from the British Film Institute (BFI) and the UK Government's Department for Digital, Culture, Media, and Sport (DCMS). Using these secondary sources, we examined specific variables relating to film consumption including age, income, location, education and cultural preferences. These secondary datasets provided a baseline of representative groups against which we could compare our three-wave survey as well as the interview and film-elicitation group data. The DCMS dataset was the 2016/17 quarter 4 sample ( $N=10,171)$ of 'Taking Part', their ongoing biannual face-toface household survey that provides data about culture, art, sport and digital engagement. It is based on a representative sample of 10,000 adults aged $16+$ who are normally resident in England (DCMS, 2017a). We compared this with the BFI's 'Opening Our Eyes' dataset (BFI, 2011, also see Northern Alliance and Ipsos MediaCT, 2011 and Hanchard, 2019), an online self-completion questionnaire $(n=2,036)$, representative of UK individuals aged 15 to 74 .

Using the statistical programming language $\mathrm{R}$ (version 3.6.1 'Action of the toes') and associated packages we carried out Latent Class Analysis (using the poLCA package version 1.4.1), Hierarchical Cluster (using the pvclust package version 2.0.0) and Regression modelling (using the glm package version 3.6.1) to uncover various relationships within the secondary datasets (see Hanchard et al., 2019; Yates et al., 2019b). In this, we looked at the clustering of film genre preferences; and which key cultural, social and economic factors best predicted both film genre preference and film genre viewing (in any format). From this, we identified a socio-cultural profile of key variables associated with film preferences and film viewing made up of education, income, age, location and perceptions of other cultural forms. This secondary analysis showed that patterns of film consumption are shaped by economic and cultural capital (Bourdieu, 1984). However, this is not strongly determined and there is also some individual choice (Bauman, 1998; Bauman, 2001), where we see a range of links between preferences and consumption of different film genres and consumers' perceptions of other cultural forms. These patterns are influenced by the socio-cultural profiles outlined above as well as levels of access at the regional scale (see the discussion of policy and industry analysis below).

Using the variables identified in the secondary data analysis, the first wave of the three-wave survey collected 5071 responses from a regionally representative sample of adults to establish patterns of film consumption in the four regions covered by BtM (see Yates et al., 2019a). This survey looked to confirm and build on the results of our secondary analysis. To do this it replicated the key measures from these datasets and in addition, included questions in response to areas identified as important in our analysis of interview data, such as:

- Engagement with a range of cultural activities - based on those in the DCMS data

- Frequency of film watching

- With whom films were watched

- How film experiences were discussed or shared

- Influences on film selection

- Influences on venue selection

- Cultural perception of film - following BFI data

- Access to and uses of the internet and digital media 
Using LCA, Hierarchical Cluster and Regression models, the first wave survey confirmed the clustering of film genre preferences we found in the secondary datasets and established a range of motivations for attending and consuming film (see Hanchard et al., 2019 and Merrington et al., 2019). Following this, the second and third waves of our three-wave survey (which gathered 547 and 250 responses respectively), we explored these patterns through time; specifically following those who were identified as engaging with 'specialised film' in the first wave. Over a period of four months these two surveys tracked the film viewing of those who had previously been identified as having consumed 'specialised film'. To do this, the survey design also drew on the findings from the interviews, and asked about the details of up to five films viewed by each respondent over the previous two months, gathering details about:

- Films watched

- How the respondent categorised each film (e.g. according to genre or other features)

- Forms of personal, intellectual and emotional engagement with each film

- Format and location the film was watched in (e.g. cinema, $\mathrm{TV}$, digital device etc.)

- Aspects liked and disliked about the film

- With whom or with which groups the film was watched, shared or discussed

Bringing the secondary data analysis together with our three-wave survey allowed us first to generate general findings about film consumption within broader cultural consumption; then to focus on film consumption across a variety of genres in relation to taste, place and social context; and finally to focus on film consumption behaviours and perceptions of those who form an 'audience' for specialised film.

These surveys identify patterns of film consumption, including patterns of preferences, ways of watching, the socio-economic and cultural backgrounds of audience members and how these influenced the ways audiences form. In bringing this together in the ontology we can start to identify the relationships between these profiles and the meaning film has for people (see the section on 'Film-elicitation groups' below), as well as the access people have to film (see the section on 'Policy and industry perspectives' below) and how they consume film (see the section on 'Audience interviews and coding' above).

\section{Film-elicitation groups}

To understand the relationship between films and the ways in which audiences interpret them, we carried out 16 film-elicitation groups - four per BtM studied region (see Forrest et al., 2019). The film-elicitation groups covered urban and rural areas, with five to six participants in each group recruited purposively via social networks and local film communities (supplemented by snowball sampling). This involved e-mailing, phoning, and/or visiting in person several local interest groups and film-related organisations. While many participants self-identified as cinephiles, others did not, with some reporting limited or no experience of accessing independent cinemas. The film-elicitation group sessions were audio-recorded and then stored on a password protected web-based cloud-storage folder. These will be deleted on the project end date (30-Sep-2020) The sessions were and conducted by Dr. David Forrest (with support from Dr. Peter Merrington in the North East and North West, Helen Rana in the South West, and Dr. Matthew Hanchard in Yorkshire and the Humber) within film-screening venues (e.g. independent and community-cinema spaces) in Newcastle and Berwick, Manchester, Bristol and Dursley (Gloucestershire), and Sheffield. In each film-elicitation group, we showed extracts from four films (each between 6 and 11 minutes long) then asked participants how they interpreted or decoded them, and how they understood the filmic stories in each extract. This is a familiar film studies methodology as an adapted version of what social scientists call photo-elicitation methods (Banks \& Zeitlyn, 2015). Although for reasons of time we were unable to show whole films, the extracts were chosen as self-contained film sequences that allowed us to explore audience engagement with specific cinematic techniques.

We selected extracts from eight films screened in independent cinemas between 2016 and 2018, choosing a mixture of foreign language and British films to encourage interpretation of both distant and more familiar cultures:

- I, Daniel Blake (Loach, 2016)

- Things to Come (Hansen-Løve, 2016)

- Call Me By Your Name (Guadagnino, 2017)

- Dark River (Barnard, 2017)

- God's Own Country (Lee, 2017)

- Loveless (Zvyagintsev, 2017)

- The Eagle Huntress (Bell, 2016)

- Happy End (Haneke, 2017)

In each film-elicitation group, participants were invited to reflect on their general experiences of film consumption before viewing the first extract. We invited further discussion immediately after showing each film extract, ensuring all participants provided input before moving on to the next extract. The questions we asked directed participants to reflect on how the film extract made them feel, and to identify anything they found significant. This aimed to provoke open and interactive discussion, and to facilitate collaborative meaning-making, offering insights into the ways in which each group constructed meanings through the narrative and aesthetic strategies of each specialised film. Our method was also designed to explore the ways in which people draw on lived experiences as resources for interpretation and reception, and the film-specific barriers and enablers for participation in specialised film. 
Overall, the film-elicitation groups allow us to contextualise nuance in the different interpretations of specialised film made by film-elicitation group members across different socio-cultural profiles, and in the diversity of meanings generated by watching such films. In this, our approach does not reduce interpretation to any specific film extract or socio-cultural profile. Instead it identifies relationships between them which shape interpretive practices. This approach deepens and extends other studies (e.g. Barker \& Brooks, 1998; Geimer, 2016) by examining a wide spectrum of films, filmic experiences, and interpretive resources. To that end, film-elicitation groups build upon textual analysis, and extend institutional and production studies, and historical approaches to film studies by foregrounding individual audience voices. Clearly, those voices are also heard through the various audience interviews we have conducted.

To qualitatively code the film-elicitation group discussions for the purposes of analysis in a suitable format for ingestion into our database, we mirrored the audience member interview coding process (see the section on 'Audience interviews and coding'). First, we used the interview coding scheme as a template, using its existing nodes and relationships as a structure. Mirroring the interview coding scheme in this way ensured that nodes and relationships represented the latest iteration of the ontology. However, we found that this scheme required some modification (changes to the existing coding scheme) because the research questions and aims of the film-elicitation group differed from those of the audience member interviews. For example, as a minor amendment, we created a new node for the film title Dark River because it did not appear within the interview coding. At other times, the coding led to more substantial changes; for example, we created a new high-level node called Interpretive Resources with various subnodes beneath it, including nodes for life experiences such as Work as a Nurse [Mental Health] and Unemployment to cover the life experiences people draw on to make sense of film content.

One of the key aspects of working with an ontology is to follow the agreed coding process consistently across all datasets to ensure meaningful comparability between them. The process should also provide enough flexibility for the coding scheme to be extended and modified during data analysis. The modifications made to our coding scheme for the purposes of the film-elicitation groups fed into further iterative revisions of the ontology.

\section{Policy and industry perspectives}

To understand the film policy and industry contexts in which film viewing takes place and to assess regional access to specialised film, we analysed industry reports and policy documents and interviewed policy makers and industry professionals (see Higson et al., 2020b). Film policy and industry practices help shape the ways in which and the extent to which audiences can access film outside the mainstream and beyond the multiplex. Questions of funding, production, distribution, exhibition and other forms of film dissemination all act as gatekeeping processes that set the parameters for film viewing. To that extent, they are central to the relationship between films and film audiences and play an important mediating role between producers and audiences (Smits et al., 2018).

To develop a better understanding of how policies and industry practices address these concerns about access and provision, we first carried out an in-depth, qualitative analysis of industry and policy reports by key public support organisations. These included the former UK Film Council (UKFC), the British Film Institute (BFI) and the DCMS in the UK, as well as European organisations like Creative Europe (for a full list see Higson et al, 2020b). The BFI, for instance, took over from the UKFC as the lead body for film in the UK in 2011, therefore we focused analysis on key strategy documents that set out their approach to increasing audiences for specialised film, including BFI Film Forever from 2012 to 2017 and BFI2022 covering 2017 to 2022 (BFI, 2012; BFI, 2017). This enabled us to understand in broad terms the patterns of funding, production, distribution and exhibition of specialised film in the UK, and to develop the industry and policy aspects of the ontology. In addition, we analysed reports by UK support organisations such as the Independent Cinema Office, the Film Distributors' Association and the British Video Association, and European support organisations such as European Audio-visual Observatory and Europa Cinemas. Such reports also included statistical data about the number of specialised films released, their box office share and release patterns, and top performing titles, which we also gathered.

To complement the document analysis, we conducted 27 elite interviews with representatives from the UK film industry and policy-makers (see Higson et al., 2020a). We asked questions about the current policies adopted by the organisations for which they worked and their approaches to film selection, programming, curation and audience development, and built on the issues raised in the document analysis from different perspectives. Interview subjects were selected according to their professional roles, levels of decision-making influence (Harvey, 2011) and experience and their location (thereby ensuring that at least some of the interviewees were active in the regions on which the research focuses). The interviews were semi-structured and coded following the same process as the film-elicitation groups. The interviewees included senior management from national and regional cinema support agencies, distributors, online platform marketing managers, film programmers and key staff from both commercial cinema chains and independent cinemas. Interviewing those primarily involved with mainstream content, such as multiplex cinema managers, allowed us to include their perspectives on the market for specialised film. Interviews were also undertaken with industry practitioners in roles relating to specialised film and cultural diversity. For instance, art-house cinemas often work with curators of special programmes to pay homage to individual directors or to draw attention to themes that are often underrepresented in society. These interviews again helped enrich our understanding of the market for specialised film.

Our experience of interviewing professionals in these positions was that their expertise was particularly helpful in situating 
their business strategies within the broader field of distribution, exhibition, access and consumption (Smits, 2016). They knew about the development of the specialised market for film in the UK over the past 10 years and were able to inform us about changes in the marketplace. Examples include the growth of online streaming subscription services and the emergence of 'boutique' chains (Picturehouse, Curzon and Everyman), which show a combination of mainstream and specialised films. We also asked exhibitors how they define audiences, who they think of as their audience (for instance, is it film specific or based on demographics, location) and what sort of research they undertake themselves to analyse the different audience groups that access their offerings. Through the ontology we can cross-reference these industry perceptions and research findings with the other data we have gathered for this project. The interviews were also useful for identifying the sort of programming initiatives that work well for specialised film, what challenges there are, what sorts of areas require improvement, the role of public support organisations in assisting specialised film, and collaborations developed between exhibitors to support specialised film.

The policy and industry expert interview insights allow us to question and deepen the knowledge gained from the document analysis and through the ontology understand the ways in which policy and industry practices shape audiences and viewing experiences. They also enable us to understand the extent to which geographical location determines the range of specialised films audiences can access, even in a world in which films are increasingly watched online.

\section{Results: An ontology in action}

In this section we explain how the ontology helps us to work across our mixed-methods data by presenting a workedthrough example. This starts with a visualisation of the ontology (Figure 1) before we present primary data and explain how the ontology helps us to identify relationships within it. For this, we use WebVOWL (version 1.1.7) to represent our ontology. WebVOWL is an interactive semantic web-based application often used to visualise ontologies (Dudáš et al., 2018). It uses the visual notation for OWL ontologies (VOWL) to structure the format of its visualisation, e.g., as standardising set of rules for the representation of classes, colours, shapes, and manor other aspects of the visualisation (Negru et al., 2014). It then applies those rules to the elements of a project in its native web ontology language (OWL). For this, VOWL visualisations are imported into WebVOWL as JSON (JavaScript Object Notation) files. To do this, we used the OWL2VOWL (version 0.3.7) conversion tool (a core feature of WebVOWL) to convert our Java-based graph database (see 'defining an ontology in mixedmethods audience studies' above) into a JSON file before importing into WebVOWL ready for visualisation. Our ontology (see Figure 3 below) can be downloaded as an .XML (extensible markup language, a subset of HTML) formatted .owl file (see Pidd et al., 2020), which can be visualised in any webbased VOWL service, such as WebVOWL or any alternative (e.g. OWLGrEd or OWLViz).

To expand on how the ontology helps us, we talk through this triple below by explaining how it relates to our mixed-methods data. By using the ontology's entities (blue circles), entity characteristics (green rectangles), and relationships (blue rectangles) set out in Figure 3 and Figure $4^{2}$ (also see the section on 'Defining an ontology in mixed methods audiences studies' above) we can explore our data in a consistent way across our datasets. For example, in Figure 4 the entity Journey is connected to another called Person through a relationship labelled isMadeBy. We can also see that the entity Person is connected to entity characteristics such as age, gender, and education and that Person is connected to another entity called Motivation through a relationship labelled hasAMotivationOf.

In another triple, Journey (the entity emphasised in Figure 4 with a red highlighted outline) is connected to Place through a relationship labelled isMadeToA. By extension, Place is connected to an entity called Location through a relationship labelled isLocatedAt. This interconnected string of triples allows us to search relationally across our data to see what sorts of journeys people make to watch film, where they go - both in terms of specific types of place (e.g. to the cinema or watching at home) and in terms of proximate locations (e.g. a specific city/town or cinema), why they choose to travel (their motivations), and what demographic composition they hold (e.g. age, education, gender). For this, the ontology allows us to see that within the qualitative interviews with audience members, people tend to travel to specific locations in order to watch films they like, that may not be available locally. In this these choose specific places to do so:

'...Important part of my life, not as much as it used to be, but I have gone to the cinema since I was very young and I continue to look out for films I like, and I travel to Edinburgh and Newcastle to see them anyway...I would say it is generally, the, I would say that I like auteur cinema. At one time I was really big fan of the European cinema....and I still like foreign films, American films...I [have] just continued to look out for well-reviewed films, and going to see them and wanting to travel more...[like] travel[ling] last night to Newcastle, I saw the Death of Stalin, which I really liked.'

Mark - male, aged 65+, retired, education undisclosed.

Here, Mark describes travelling from their home (both a Place) in Berwick-upon-Tweed to Edinburgh and Newcastle (both a Location), in order to watch the films they like (a Motivation) - namely auteur cinema, European cinema, and foreign and American films. They also name the last film they saw, and explain that they have been going to the cinema since a very young age. Following the triples identified in Figure 4, Jessie explains that her husband holds a similar sentiment:

'...my husband is very fond of Studio Ghibli, and we have been known to go to London just to see a Studio Ghibli film...I'm probably more likely to go to, like, Glasgow Film Festival or Edinburgh Film Festival to have a binge...Keswick is just a bit far for me to go just for one film...'

${ }^{2}$ The red pins on each entity are not significant. They are a feature of WebVOWL that allows elements to be 'pinned' together for easy visualisation. 


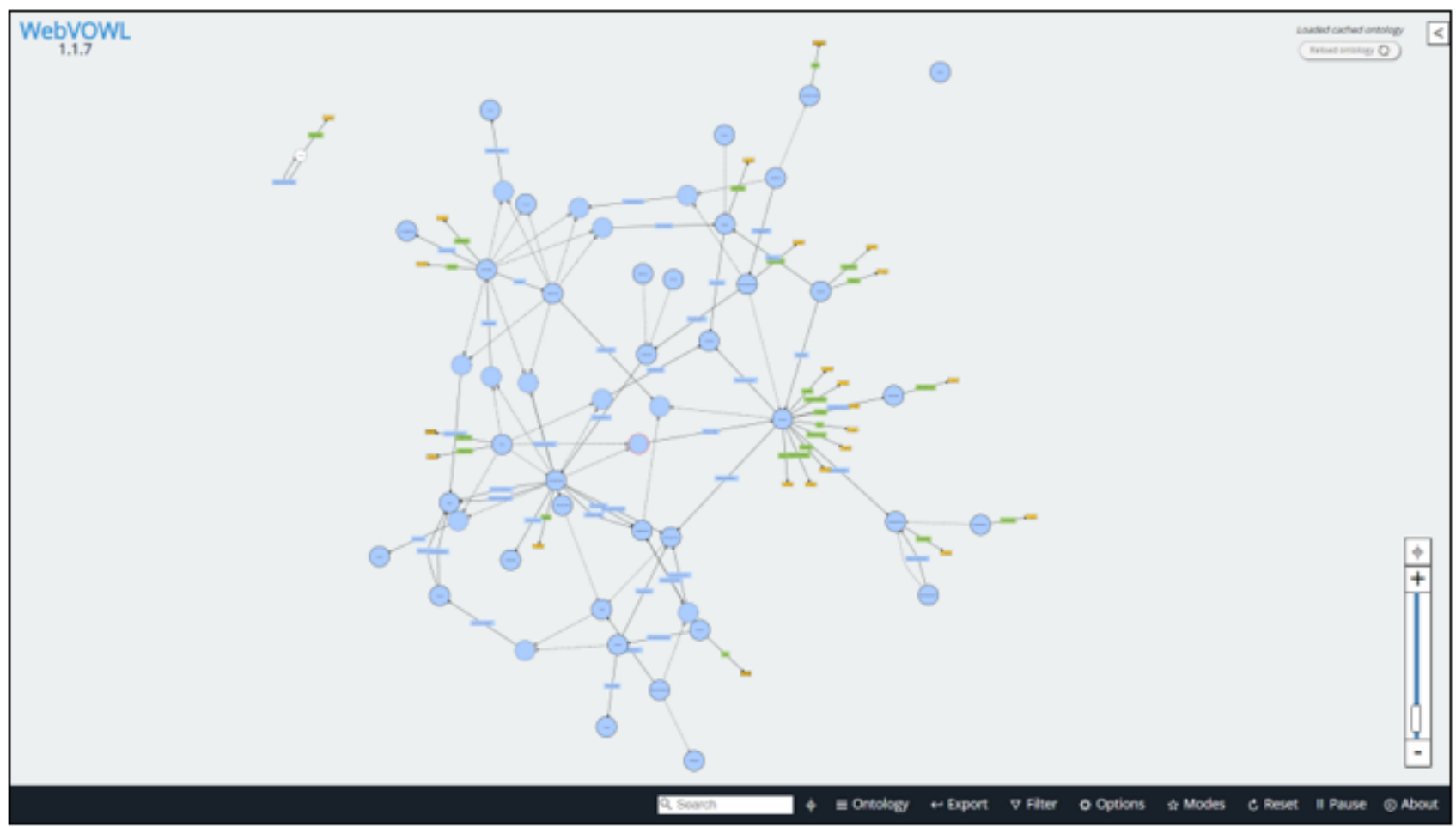

Figure 3. Visualisation of our ontology in WebVOWL.

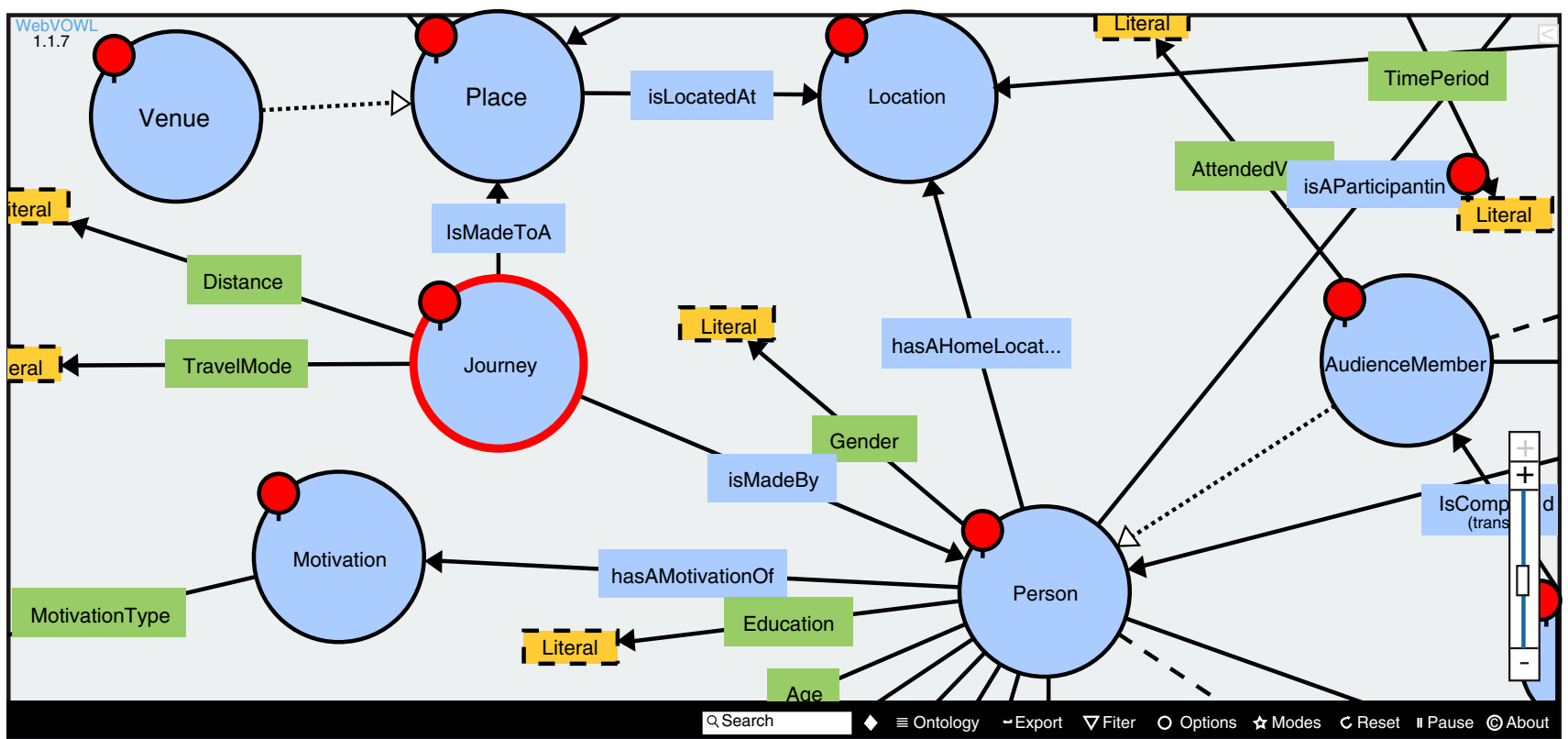

Figure 4. WebVOWL visualisation of our ontology.

Jessie - female, aged 65+, works in the creative industries on self-employed basis, and holds qualifications above A-Level.

She describes traveling to London (a Journey to a Location) with him (both listed as a Person) in order to watch the specific type of film he likes (a Motivation). She also describes travelling to Glasgow and Edinburgh (both a Location) to watch films at film festivals (a specific type of Place) in order to binge-watch the films she likes (her Motivation). By contrast, when Jessie she explains that although Keswick (a Location) is closer to her home-town (Carlisle) at only one 
hour away by car, she notes that she would only be able to watch one film there rather than being able to binge-watch (as she could at a film festival). As such, she has no Motivation for making the Journey there to do so.

Because we gathered demographic details about each interview participant (see Wessels et al., 2019) we can also search for similar patterns within the survey data (see Yates et al., 2019a) and to see what sorts motivations different people have for travelling to watch films at the cinema by exploring the extent to which the Location of a venue affects people's choice to watch (their Motivation) at the cinema (a Place). For example, the below clustered bar chart (generated from the survey wave 1 data in IBM SPSS statistics version 26) shows that $30 \%$ $(n=1533)$ and $32 \% \quad(n=1624)$ of the survey respondents find that the convenience of having a cinema located close to their home (a Motivation) affects their decision to go there to watch films a little or lot respectively (62\% in total), compared with $6 \%(\mathrm{n}=295)$ who said it did not affect their decision much, or $8 \%(n=406)$ that said it did not affect their decision at all.

As such, we can see that the motivations that Mark and Jessie have for travelling to watch film are not typical across the data - but they are still relatively important beyond those two participants given that people in the 55+ age group (which both Mark and Jessie fall within) are proportionately more likely to have stated 'not at all' to this survey question (see Figure 5).
What the ontology does then, is allow us to see that people have specific motivations for seeing film such as watching the films they like at a specific cinema or event, and that they will travel to watch them if they are not available locally. However, age appears to be a key factor that affects their likelihood of travelling to see films. Within this, a majority of people (of all ages) find the convenience of having a cinema located close to their home a key deciding factor in their motivation for going to watch a film there. However, a proportionally higher number for people aged over 55+ are likely to state 'not at all' when asked if having a cinema nearby is a key factor in their motivation for watching there. In this, the string of triples discussed above highlights a set of relationships between the data. The ontology automates the identification of relations between various datasets that might not have been otherwise have been uncovered through traditional mixed-methods research alone. We can then explore those relationships either through standalone analyses, or via data visualisations. That is, while the same set of findings could be derived through a manual process of combining traditional qualitative and quantitative analyses, the ontology automates that task, and as such combines them consistently at a scale and pace that would be infeasible for even a very well-resourced research team to do.

\section{Conclusion}

The ontological approach provides us with a conceptual model of the knowledge domain of film audiences that considers and analyses the evidence contained in several diverse datasets.

Q10: How much do the following sources influence you when choosing a film to watch at the cinema?

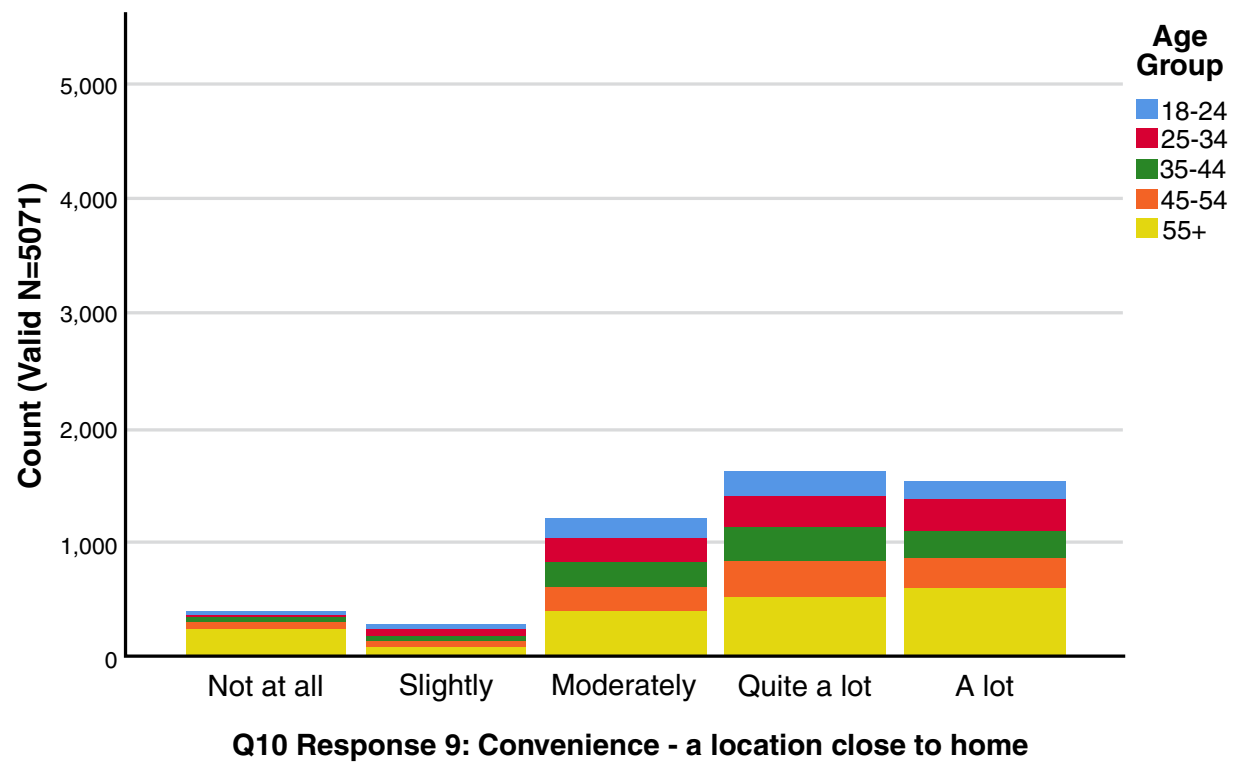

Figure 5. A clustered bar chart of survey wave one question 10 response 9 filtered by age group. 
However, it does not resolve the challenge of creating a conceptual model. Any database requires some definition of what data it will contain. The challenge here is twofold: to reconcile very different datasets, both quantitative and qualitative; and to process the data in ways which comply with the requirements of the database without distorting the data in the cause of standardising or categorising it. For qualitative data, such as interview transcripts, this requires a considerable amount of analysis and interpretation of the data so that ontologically significant content can be manually coded to align with the emerging ontological model. This becomes a symbiotic process whereby further development of the ontology is informed by researcher's discovery and encoding of content that is considered meaningful to the research. As such, it is an iterative process. At the outset of the project, the purpose of the ontology was to enable us to create a unified view of the data that would facilitate sophisticated interrogation later on (e.g. querying and visualisation), but the coding requirements of the transcripts and policy documents have shown that an ontology provides a more immediate and useful purpose as a conceptual framework to guide the initial deep reading of the data. In other words, the ontology evolves from a knowledge domain data model into a conceptualisation of film worlds.

This paper has set out an approach to capturing and understanding the richness of film audience experiences in the context of changing cultural provision, access and competition for audience attention. Here we have presented the potential of using a mixed-methods and ontology approach that enables a complex range of data to be consistently questioned in order to develop richly nuanced and meaningful insights into film audiences at a scale, depth and complexity that has hitherto been lacking. Adopting an ontology to keep all of this data in perspective is enabling us to produce a representation of film worlds that is genuinely much greater than the sum of its parts, and much greater in scope than any other similar project that we are aware of. By adopting this approach, we can explore how specialised film engagement relates to cultural engagement in general, how audiences engage with a range of different types of film through online platforms and venues and how they make meaning from their experiences. This approach also allows us to assess the extent to which audience activities and experiences are shaped by national and regional film policies, industry practices and the decisions of cultural intermediaries. Its impact will include facilitating informed and critical engagement with debates about the role of policy and public funding in enabling a more diverse film culture. As well as understanding the cultural value of a diverse film culture more generally. This will be encouraged by making the ontology, the data, and a series of data analytical tools openly accessible to film development stakeholders for use in their own work.

\section{Ethics and consent}

The project involves ethical issues regarding research on human subjects in film-elicitation groups, interviews, survey and Delphi workshops and in the collection, treatment and storage of data. All research activity abides by Universities UK's Concordat to Support Research Integrity and the Arts and
Humanities Research Council (AHRC) ethical expectations based on the Research Council guidelines including informed consent. The proposal has been assessed and approved by Newcastle University Research Ethics review (reference: BH161701) - see details at: http://www.ncl.ac.uk/res/research/ethics_governance/ethics_governance_toolkit/ethics/index.htm

Throughout the research full attention has been paid to informed consent, confidentiality, anonymity, avoidance of harm, data ethics, and ensuring that participation was voluntary. Information sheets and consent forms were explained verbally, so that participants could ask questions. The film elicitation film-elicitation groups did not use 'upsetting' films, with the proviso that if a participant found a film emotionally upsetting/distressing, then that person could leave. Managing confidentiality was more difficult in elite interviews with industry and policy participants where they may be more easily identified. For this, we allowed each participant to decide whether their interview would be 'on the record' or not.

\section{Data availability}

\section{Source data}

DCMS Survey. One dataset used in this paper is composed of responses to DCMS survey release for quarter 4 of their longitudinal survey titled 'Taking Part' (see DCMS (2017a), available at: https://www.gov.uk/government/statistics/taking-part-201617quarter-4-statistical-release (accessed 30-Jan-2020) under the Open Government License agreement.

BFI survey. One dataset used in this paper is composed of responses to a British Film Institute (BFI) survey titled: 'Cultural Consumption' conducted by IpsosMORI in 2011. The BFI provide the survey dataset as appendix 4 of their larger report:

Northern Alliance and Ipsos MediaCT (2011) Opening our eyes: How film contributes to the culture of the UK (Report), London: British Film Institute. Available at: https://www.bfi.org. uk/about-bfi/policy-strategy/opening-our-eyes-how-film-contributes-culture-uk.

Duplicate copies of the dataset (without attributed DOI references) are available via both the Digital Humanities Institute (DHI) data repository at the University of Sheffield: https://www. dhi.ac.uk/san/btm/Data_122018/Cultural\%20Contribution.zip and direct via the BFI website: http://old.bfi.org.uk/publications/ openingoureyes/downloads/Appendix-4-Cultural-ContributionSurvey-Data-SPSS-Format.zip.

A copy of the BFI dataset has been archived using the Enlighten research data repository managed by the University of Glasgow.

Enlighten: Opening our eyes: how film contributes to the culture of the UK - Appendix 4: Cultural consumption survey data. http:// dx.doi.org/10.5525/gla.researchdata.853 (Hanchard, 2019)

This project contains the following data:

- Cultural_Contribution.zip (Zip file containing BFI Opening our eyes survey data) 
The University of Glasgow was granted full permission to store the dataset and to make fully it open access for public re-use without restriction. This permission was granted by the British Film Institute (BFI) Research and Statistics Manager on 13-Aug-2019.

\section{Underlying data}

All source data discussed is this paper are from the Enlighten research data repository, managed by the University of Glasgow:

Enlighten: Research Data: Beyond the Multiplex - Audience Member Interview. http://dx.doi.org/10.5525/gla.researchdata.883 (Wessels et al., 2019)

This project contains the following underlying data:

- Transcripts.zip (copy of all anonymised audience member interview transcripts)

- Coding_Scheme.docx (NVivo coding scheme)

- Classification_sheet.xlsx (classification sheet with participant demographic details)

Enlighten: Research Data: Beyond the Multiplex - Audience Member Interview. http://dx.doi.org/10.5525/gla.researchdata.883 (see Yates et al., 2019b)

This project contains the following underlying data:

- Responses_Wave_1.xlsx (Questions and responses from Wave 1)

- Responses_Wave_2.xlsx (Questions and responses from Wave 2)

- Responses_Wave_3.xlsx (Questions and responses from Wave 3)

Enlighten: Research Data: Beyond the Multiplex - Film Elicitation. http://dx.doi.org/10.5525/gla.researchdata.885 (see Forrest et al., 2019)

This project contains the following underlying data:

- groups.zip (anonymised film-elicitation group transcripts)

- classification_sheet.xlsx (classification sheet with participant demographic details)

- Coding_Scheme.docx (NVivo coding scheme)
Enlighten: Research Data: Beyond the Multiplex - Expert interview. http://dx.doi.org/10.5525/gla.researchdata.945 (see Higson et al., 2020a)

This project contains the following underlying data:

- Transcripts.zip (anonymised film-elicitation group transcripts)

- Questions.docx (Questions used in interviews)

- Coding_Scheme.docx (NVivo coding scheme)

Enlighten: Research Data: Beyond the Multiplex - Policy and Industry document analysis. http://dx.doi.org/10.5525/gla.researchdata.942 (see Higson et al., 2020b)

This project contains the following underlying data:

- List_of_documents.docx (list of all analysed policy and industry documents)

- Coding_Scheme.docx (NVivo coding scheme)

\section{Extended data}

Enlighten: Research Data: Beyond the Multiplex - Ontology. http:// dx.doi.org/10.5525/gla.researchdata.957 (Pidd et al., 2020)

This project contains the following extended data:

- Beyond\%20the\%20Multiplex\%20-\%20Computational\%20 Ontology\%20-\%20Version\%201.owl (copy of our computational ontology as visualised in Figure 3 and Figure 4)

This file can be downloaded as a .owl file from the above doi, and accessed as XML (extensible markup language) in any web browser or opened with any OWL visualisation platform, such as WebVOWL, OWLGrEd, or OWLViz.

All data listed above are, unless stated otherwise, available under the terms of the Creative Commons Attribution 4.0 International license (CC-BY 4.0).

\section{Software availability}

To develop a socio-cultural index from these datasets, we used a free statistical software available under a GNU Affero general public license (AGPL), called R. R Studio, the graphical user interface used to operate $\mathrm{R}$ beyond its command-line interface is also freely available under a GNU AGP licence. The $\mathrm{R}$ packages used within this paper (poLCA version 1.4.1, pvclust version 2.0.0, glm version 3.6.1, and RcmndrMisc version 2.5.1) are $\mathrm{R}$ libraries, and as such they are also openly available under R's GNU AGPL. R, R Studio, and all packages named above can be downloaded directly from the R project website: https://www.r-project.org/.

\section{References}

Arts Council England: Arts Audiences: Insight. London: Arts Council England. 2011 Reference Source

Banks M, Zeitlyn D: Visual Methods in Social Research. London: Sage. 2015. Reference Source

Barker M, Brooks K: Knowing Audiences: 'Judge Dredd', its friends, fans and foes. Luton: University of Luton Press. 1998. Reference Source

Barker M, Mathijs E: Research World Audiences: The experience of a complex Methodology. Participations. 2012; 9(2): 664-689.

Reference Source 
Barnard C: Dark River. United Kingdom: Arrow Films. 2017

Reference Source

Bauman Z: Freedom. Milton Keynes: Open University Press. 1998. Reference Source

Bauman Z: The Individualized Society. Cambridge: Polity. 2001. Reference Source

Becker H: Art Worlds. Berkeley: University of Berkeley Press. 1982. Reference Source

Bell O: The Eagle Huntress. Mongolia: Altitude Film Distribution. 2016

Reference Source

BFI: Opening our eyes: how film contributes to the culture of the UK. Appendix 4: Cultural consumption survey data [dataset], London: BFI. 2011. (accessed 30Jan-2020).

Reference Source

BFI: Film Forever: Supporting UK Film - BFI Plan 2012-2017. London: BFI. 2012. Reference Source

BFI: BFI2022: Supporting UK Film - BFI Plan 2017-2022. London: BFI. 2017. Reference Source

Bourdieu P: Distinction: a social critique of the judgement of taste. Abingdon: Routledge. 1984.

Reference Source

Charmaz K: Teaching Theory Construction With Initial Grounded Theory Tools: A Reflection on Lessons and Learning. Qual Health Res. 2015; 25(12) 1610-1622.

PubMed Abstract | Publisher Full Text

Christie I: Introduction: in search of audiences. In Christie, I. (ed.) Audiences: Defining and Researching Screen Entertainment Reception. Amsterdam: Amsterdam University Press. 2012; 11-24.

Publisher Full Tex

Corbett S, Wessels B: Active audiences and reflexivity: How film audiences form in northern English regions. Participations. 2017; 14(2): 1-17. Reference Source

Cresswell J: Research Design: Qualitative, Quantitative and Mixed Methods Approaches. Los Angeles. Sage. 2009.

Reference Source

Crossley N, Edwards G: Cases, Mechanisms and the Real: The Theory and Methodology of Mixed-Method Social Network Analysis. Sociol Res Online. 2016; 21(2): 217-285.

Publisher Full Text

DCMS: 'Taking Part Survey: England Adult Report, 2016/17 - Statistical release quarter 4' [dataset]. DCMS/Crown: London. 2017a; (accessed 30-Jan-2020). Reference Source

DCMS: Taking Part Survey: England Adult Report findings, 2016/17. DCMS/ Crown: London, 2017b; 1-35.

Reference Source

Dudáš M, Lohmann S, Svátek V, et al:: Ontology visualization methods and tools: A survey of the state of the art. The Knowledge Engineering Review. 2018; 33: e10.

Publisher Full Text

Evans E: Transmedia Television: Audiences, New Media, and Daily Life. Abingdon: Routledge. 2011

Publisher Full Text

Forrest D, Merrington P, Hanchard M et al: 'Beyond the Multiplex - Filmelicitation groups' [Data Collection]. Enlighten data repository. 2019.

http://www.doi.org/10.5525/gla.researchdata.885

Geimer A: Preferred Readings and Dissociative Appropriations: Group Discussions Following and Challenging the Tradition of Cultural Studies. In Reinhard, C. and CarrieLynn, O. (eds) Making Sense of Cinema: Empirical Studies into Film Spectators and Spectatorship. London, 2016; 77-96.

Publisher Full Text

Guadagnino L, (Director): Call Me by Your Name [Motion Picture]. France:

Memento Films. 2017.

Reference Source

Hanchard M: copy of BFI 'Opening our eyes: how film contributes to the culture of the UK - Appendix 4: Cultural consumption survey data'. [Data Collection]

Enlighten Data Repository. 2019.

http://www.doi.org/10.5525/gla.researchdata.853

Hanchard M, Merrington $\mathrm{P}$, Wessels $\mathrm{B}$, et al:: Exploring contemporary patterns of cultural consumption: offline and online film watching in the UK [version 1; peer review: 2 approved] . Emerald Open Res. 2019; 1: 16. (accessed 30-Jan-2020).

Publisher Full Text
Haneke M, (Director): Happy End [Motion Picture]. France: Artificial Eye. 2017. Reference Source

Hansen-Løve M, (Director): Things to Come [L'Avenir] [Motion Picture]. France: CG Cinéma. 2016.

Reference Source

Harvey W: Strategies for conducting elite interviews. Qual Res. 2011; 11(4)

$431-441$.

Publisher Full Text

Higson A, Townsend N, Smits R, et al:: Beyond the Multiplex - Expert interviews. Data Collection], Enlighten data repository. 2020a.

http://www.doi.org/10.5525/gla.researchdata.945

Higson A, Townsend N, Smits R, et al:: Beyond the Multiplex - Policy and Industry document analysis. [Data Collection], Enlighten data repository. 2020b. http://www.doi.org/10.5525/gla.researchdata.942

Johanson K, Glow H: A virtuous circle: the positive evaluation phenomenon in arts audience research. Participations. 2015; 12(1): 254-270.

Reference Source

Lee F, (Director): God's Own Country. [Motion Picture] United Kingdom:

Picturehouse Entertainment. 2017.

Reference Source

Livingstone S: Audience research at the crossroads: The 'implied audience' in media and cultural theory. European Journal of Cultural Studies. 1998; 1(2): 193-217. Publisher Full Text

Livingstone S: 'Audiences in an age of datafication: Critical questions for media research'. Television and new media. 2018; 20(2): 170-183.

Publisher Full Text

Loach K, (Director): I, Daniel Blake. [Motion Picture]. United Kingdom:

Entertainment One. 2016

Reference Source

Merrington $\mathrm{P}$, Hanchard M, Wessels $\mathrm{B}$, et al:: Using mixed-methods, a data model and a computational ontology in film audience research. Cultural Trends. 2019;

28: (2-3): 118-131.

Publisher Full Text

Negru S, Lohmann S, Hagg F: 'VOWL: Visual Notation for OWL Ontologies

- Specification of Version 2.0'. VOWL. (accessed 30-Jan-2020), 2014.

Reference Source

Northern Alliance and Ipsos MediaCT: 'Opening our eyes'. Polity. 2011.

Pidd M, Rogers K: Why Use an Ontology? Mixed Methods Produce Mixed Data. 2018; (accessed: 15-January-2020).

Reference Source

Pidd M, Rogers K, Hanchard M, et al.: Beyond the Multiplex: Ontology. [Data Collection] Enlighten: Research Data. 2020;

http://www.doi.org/10.5525/gla.researchdata.957

Saldana J: The Coding Manual for Qualitative Researchers. London: Sage

Publications. 2012.

Reference Source

Silverman D: Doing qualitative research: a practical handbook. London: Sage. 2010.

Reference Source

Smits R: Gatekeeping and Networking Arrangements: Dutch Distributors in the Film Distribution Business. Poetics. 2016; 58: 29-42.

Publisher Full Text

Smits R, Higson A, Mateer J, et al:: Distributing Films Online. J Br Cine Telev. 2018; 15(2): 291-299.

Publisher Full Text

UKRI: Beyond the Multiplex: Audiences for Specialised Film in English Regions. UK Research and Innovation. 2017; (Accessed: 13 December 2018). Reference Source

Wessels B, Merrington P, Hanchard M, et al:: Beyond the Multiplex - Audience Member Interview. [Data Collection] Enlighten: Research Data. 2019.

http://www.doi.org/10.5525/gla.researchdata.883

Yates S, Hanchard M, Merrington P, et al:: Beyond the Multiplex - Three-wave survey. [Data Collection] Enlighten: Research Data. 2019a.

http://www.doi.org/10.5525/gla.researchdata.884

Yates S, Wessels B, Higson A, et al.: Beyond the Multiplex: R script for Latent

Class Analyses. [Data Collection] Enlighten: Research Data. 2019b.

http://www.doi.org/10.5525/gla.researchdata.879

Zvyagintsev A: Loveless [Nelyubov]. [Motion Picture] Russia: Altitude Film

Distribution. 2017.

Reference Source 


\section{Open Peer Review}

\section{Current Peer Review Status:}

\section{Version 1}

Reviewer Report 30 March 2020

https://doi.org/10.21956/emeraldopenres.14535.r26694

(C) 2020 O'Sullivan J. This is an open access peer review report distributed under the terms of the Creative Commons Attribution License, which permits unrestricted use, distribution, and reproduction in any medium, provided the original work is properly cited.

\section{James O'Sullivan}

College of Arts, Celtic Studies and Social Sciences, University College Cork, Cork, Ireland

This is a strong article that presents a novel approach to audience analysis and a good example of what robust interdisciplinary research should look like. I have no reservations in recommending this piece for passing peer review.

The article is appropriately contextualised with the problem, methodology and significance of the findings addressed in clear and considerable detail. I particularly like how the article manages to treat expert topics in an intuitive manner without turning the overall paper into an introductory text.

The Ontology in Action section is particularly compelling and makes a significant original contribution to the field.

I have no criticisms to offer this paper.

Is the rationale for developing the new method (or application) clearly explained? Yes

Is the description of the method technically sound?

Yes

Are sufficient details provided to allow replication of the method development and its use by others?

Yes

If any results are presented, are all the source data underlying the results available to ensure full reproducibility?

Yes 
Are the conclusions about the method and its performance adequately supported by the findings presented in the article?

Yes

Is the argument information presented in such a way that it can be understood by a nonacademic audience?

Yes

Does the piece present solutions to actual real world challenges?

Yes

Is real-world evidence provided to support any conclusions made?

Yes

Could any solutions being offered be effectively implemented in practice?

Yes

Competing Interests: No competing interests were disclosed.

I confirm that I have read this submission and believe that I have an appropriate level of expertise to confirm that it is of an acceptable scientific standard.

Reviewer Report 25 February 2020

https://doi.org/10.21956/emeraldopenres.14535.r26627

(c) 2020 Treveri Gennari D. This is an open access peer review report distributed under the terms of the Creative Commons Attribution License, which permits unrestricted use, distribution, and reproduction in any medium, provided the original work is properly cited.

\section{Daniela Treveri Gennari}

Oxford Brookes University, Oxford, United Kingdom

This article present a rigorous research and an extremely important contribution to the study of audiences in the UK and beyond. The methodology has been explained with great clarity and very meticulous approach has been employed in order to put together a very complex analytical project.

The development of the ontology is of extreme importance, as it can offer a new way of analyzing different datasets (both qualitative and quantitative) and produce innovative results, a new analytical tool and data visualizations that can be of great interest for both academics and stakeholders.

While the article presents sufficient details about its methods and analysis, I believe that in order to allow replication of the method development and its use by others might need further clarifications, especially if the researchers interested in adopting this methodology are unfamiliar 
with the ontology concept. This does not certainly diminish the importance of this article. It is more a requests for further publications where scholars can successfully take advantage of this valuable tool.

Is the rationale for developing the new method (or application) clearly explained?

Yes

Is the description of the method technically sound?

Yes

Are sufficient details provided to allow replication of the method development and its use by others?

Partly

If any results are presented, are all the source data underlying the results available to ensure full reproducibility?

Yes

Are the conclusions about the method and its performance adequately supported by the findings presented in the article?

Yes

Is the argument information presented in such a way that it can be understood by a nonacademic audience?

Yes

Does the piece present solutions to actual real world challenges?

Yes

Is real-world evidence provided to support any conclusions made?

Yes

Could any solutions being offered be effectively implemented in practice?

Yes

Competing Interests: No competing interests were disclosed.

Reviewer Expertise: Cinema studies, Audience Research

I confirm that I have read this submission and believe that I have an appropriate level of expertise to confirm that it is of an acceptable scientific standard. 